\title{
Effective computation of Picard groups and Brauer-Manin obstructions of degree two K 3 surfaces over number fields
}

\author{
Hassett, Brendan ; Kresch, Andrew ; Tschinkel, Yuri
}

\begin{abstract}
Using the Kuga-Satake correspondence we provide an effective algorithm for the computation of the Picard and Brauer groups of K 3 surfaces of degree 2 over number fields.
\end{abstract}

DOI: https://doi.org/10.1007/s12215-013-0116-8

Posted at the Zurich Open Repository and Archive, University of Zurich ZORA URL: https://doi.org/10.5167/uzh-86024

Journal Article

Published Version

Originally published at:

Hassett, Brendan; Kresch, Andrew; Tschinkel, Yuri (2013). Effective computation of Picard groups and BrauerManin obstructions of degree two K 3 surfaces over number fields. Rendiconti del Circolo Matematico di Palermo, 62(1):137-151.

DOI: https://doi.org/10.1007/s12215-013-0116-8 


\title{
Effective computation of Picard groups and Brauer-Manin obstructions of degree two $K 3$ surfaces over number fields
}

\author{
Brendan Hassett • Andrew Kresch • Yuri Tschinkel
}

Received: 17 October 2012 / Accepted: 22 October 2012 / Published online: 15 February 2013

(C) Springer-Verlag Italia 2013

\begin{abstract}
Using the Kuga-Satake correspondence we provide an effective algorithm for the computation of the Picard and Brauer groups of $K 3$ surfaces of degree 2 over number fields.
\end{abstract}

Keywords Brauer groups $\cdot K_{3}$ surface $\cdot$ Effective computation

Mathematics Subject Classification $\quad 14 \mathrm{~J} 28 \cdot 14 \mathrm{~F} 22 \cdot 11 \mathrm{G} 18 \cdot 14 \mathrm{G} 25$

\section{Introduction}

Let $X$ be a smooth projective variety over a number field $k$ and $\operatorname{Br}(X)$ its Brauer group. The quotient $\operatorname{Br}(X) / \operatorname{Br}(k)$ plays an important role in the study of arithmetic properties of $X$. Its effective computation is possible in certain cases, for example: when $X$ is a geometrically rational surface (see, e.g., [37]), a Fano variety of dimension at most 3 [34], or a diagonal $K 3$ surface over $\mathbb{Q}[24,35,66]$.

Skorobogatov and Zarhin proved the finiteness of $\operatorname{Br}(X) / \operatorname{Br}(k)$ when $X$ is a $K 3$ surface [65]. Letting $X_{\bar{k}}$ denote $X \times_{\operatorname{Spec} k} \operatorname{Spec} \bar{k}$, where $\bar{k}$ is an algebraic closure of $k$, there is the

The first author was supported by NSF grants 0901645 and 0968349 . The second author was supported by the SNF. The third author was supported by NSF grants 0739380,0901777 , and 0968349.

B. Hassett

Department of Mathematics, Rice University, Houston, TX 77005, USA

e-mail: hassett@rice.edu

A. Kresch $(\varangle)$

Institut für Mathematik, Universität Zürich, Winterthurerstrasse 190, 8057 Zürich, Switzerland e-mail: andrew.kresch@math.uzh.ch

Y. Tschinkel

Courant Institute, 251 Mercer Street, New York, NY 10012, USA

e-mail: tschinkel@cims.nyu.edu 
natural map

$$
\operatorname{Br}(X) \rightarrow \operatorname{Br}\left(X_{\bar{k}}\right)
$$

Its kernel is known as the algebraic Brauer group. The quotient of the algebraic Brauer group by the image in $\operatorname{Br}(X)$ of $\operatorname{Br}(k)$ is a finite group, and may be identified with the Galois cohomology

$$
H^{1}\left(\operatorname{Gal}(\bar{k} / k), \operatorname{Pic}\left(X_{\bar{k}}\right)\right)
$$

Knowledge of $\operatorname{Pic}\left(X_{\bar{k}}\right)$ is essential to its computation. A first goal of this paper is the effective computation of $\operatorname{Pic}\left(X_{\bar{k}}\right)$ when $X$ is a $K 3$ surface of degree 2 over a number field. Special cases and examples have been treated previously by, e.g., van Luijk [41], while a more general treatment, that is however conditional on the Hodge conjecture, appears in [11].

The image of (1.1) is contained in the invariant subgroup

$$
\operatorname{Br}\left(X_{\bar{k}}\right)^{\operatorname{Gal}(\bar{k} / k)} .
$$

The finiteness of this invariant subgroup is one of the main results of [65], yet the proof does not yield an effective bound. In this paper we give an effective bound for the order of the group $\operatorname{Br}(X) / \operatorname{Br}(k)$. Combined with the results in [35], this permits the effective computation of the subset

$$
X\left(\mathbb{A}_{k}\right)^{\operatorname{Br}(X)} \subseteq X\left(\mathbb{A}_{k}\right)
$$

of Brauer-Manin unobstructed adelic points of $X$. Examples of computations of Brauer-Manin obstructions on $K 3$ surfaces can be found in $[10,20,23,64,69]$. The results here, combined with results in [12], imply as well an effective bound for the order of $\operatorname{Br}\left(X_{\bar{k}}\right)^{\operatorname{Gal}(\bar{k} / k)}$.

The finiteness results of [65] are based on the Kuga-Satake construction, which associates an abelian variety of dimension $2^{19}$ to a given $K 3$ surface and relates their cohomology, together with the Tate conjecture for abelian varieties, proved by Faltings [15]. The KugaSatake correspondence is conjectured to be given by an algebraic correspondence, but this is proved only in some special cases, e.g., [18,26,63]. Assuming this (in some effective form), one could apply effective versions of Faltings' results, obtained by Masser and Wüstholz [42-46] (see also [9]). Lacking this, we treat the transcendental construction directly, showing that computations to bounded precision can replace an algebraic correspondence, in practice.

The construction proceeds in several steps. First of all, rigidity allows us to construct the Kuga-Satake morphism between moduli spaces (of $K 3$ surfaces with polarization and level structure on one side and polarized abelian varieties with level structure on the other) algebraically over a number field, at least up to an explicit finite list of possibilities. This allows us to identify an abelian variety corresponding to a $K 3$ surface, and its field of definition. For the computation of the induced map on homology we work with integer coefficients and simplicial complexes, and computations up to bounded precision suffice to determine all the necessary maps. Then we follow the proof in Section 4 of [65] and obtain the following result.

Theorem 1 Let $k$ be a number field and $X$ a K3 surface of degree 2 over $k$, given by an explicit equation. Then there is an effective bound on the order of $\operatorname{Br}(X) / \operatorname{Br}(k)$.

In fact, we provide an effective bound on $\operatorname{Br}(X) / \operatorname{Br}(k)$ when $X$ has a primitive ample line bundle of arbitrary degree $2 d$ provided that there is an effective construction of the moduli space of primitively quasi-polarized $K 3$ surfaces of degree $2 d$ (see Definition 16). For $d=1$ this is known, via effective geometric invariant theory (see Remark 9). 
The first step of the proof is, as mentioned above, the effective computation of the Galois module $\operatorname{Pic}\left(X_{\bar{k}}\right)$. Since this is finitely generated and torsion-free, this permits the effective computation of the Galois cohomology group (1.2). Hence the proof of Theorem 1 is reduced to effectively bounding the image of (1.1).

\section{Effective algebraic geometry}

We work over an algebraic number field $k$ and denote by $k$ an algebraic closure of $k$. The term variety refers to a geometrically integral separated scheme of finite type over $k$. We say that a quasiprojective variety or scheme $X$ is given by explicit equations if homogeneous equations are supplied defining a scheme in a projective space $\mathbb{P}^{M}$ for some $M$ and a closed subscheme whose complement is $X$. By convention $\mathcal{O}_{X}(1)$ will denote the restriction of $\mathcal{O}_{\mathbb{P}^{M}}(1)$ to $X$. The base-change of $X$ to a field extension $k^{\prime}$ of $k$ will be denoted $X_{k^{\prime}}$.

Lemma 2 Let $X$ be a quasiprojective scheme, given by explicit equations. Let $f: Y \rightarrow X$ be a finite morphism, given by explicit equations on affine charts. Then we may effectively determine integers $n$ and $N$ and an embedding $Y \rightarrow X \times \mathbb{P}^{N}$, such that $f$ is the composite of projection to $X$ with the embedding and the pullback of $\mathcal{O}_{\mathbb{P}^{N}}(1)$ to $X \times \mathbb{P}^{N}$ restricts to $f^{*} \mathcal{O}_{X}(n)$. In particular, $f^{*} \mathcal{O}_{X}(n+1)$ is very ample on $Y$ and we may obtain explicit equations for $Y$ as a quasiprojective scheme.

Proof We may without loss of generality suppose that $X$ is an open subscheme of a projective space, $X=\mathbb{P}^{M} \backslash Z$, with $f$ given by explicit equations over affine charts of $X$, each the complement in $\mathbb{P}^{M}$ of a divisor containing $Z$. Specifically, the coordinate ring of each corresponding affine chart of $Y$ is presented as an algebra over that of $X$ by means of explicit generators and relations. We may determine, effectively, an integer $n$ such that each generator extends to a section of $\mathcal{O}_{X}(n)$. Then for suitable $N$ we have an embedding $Y \rightarrow X \times \mathbb{P}^{N}$ satisfying the desired conditions.

We collect effectivity results that we will be using freely.

- Hilbert schemes: Given $d, n$, and $N$, there are only finitely many possible Hilbert polynomials of purely $n$-dimensional degree- $d$ reduced closed subschemes of $\mathbb{P}_{k}^{N}$; cf. [29, Corollary 6.11], and [21] for an effective version. So the space of such schemes, and also of bounded maps between such schemes (as represented by the graph, with a bound applied to the degree of the graph in the product of ambient projective spaces under the Segre embedding), may be constructed effectively as a disjoint union over the possible Hilbert polynomials of open subschemes of the respective Hilbert schemes.

- Effective normalization in a finite function field extension: Given a quasiprojective variety $X$ over $k$, presented by means of explicit equations, and another algebraic variety $V$ with generically finite morphism $V \rightarrow X$ given by explicit equations on affine charts, to compute effectively the normalization $Y$ of $X$ in $k(V)$, with finite morphism $Y \rightarrow X$. See [47] and references therein.

- A form of effective resolution of singularities: Given a nonsingular quasiprojective variety $X^{\circ}$ over $k$, to produce a nonsingular projective variety $X$ and open immersion $X^{\circ} \rightarrow$ $X$, such that $X \backslash X^{\circ}$ is a simple normal crossings divisor. This follows by standard formulations of Hironaka resolution theorems, for which effective versions are available; see, e.g., [6].

- Effective invariant theory for actions of projective varieties: Given a projective variety $X$ and a linearized action of a reductive algebraic group $G$ on $X$ for $L=\mathcal{O}_{X}(1)$, to compute 
effectively the semistable locus $X^{s s}(L)$ and stable locus $X^{s}(L)$ of geometric invariant theory (GIT), the GIT quotient variety $X / / G$, open subset $U \subset X / / G$ corresponding to $X^{s}(L)$, and quotient morphisms $X^{s s}(L) \rightarrow X / / G$ and $X^{s}(L) \rightarrow U$. This is standard, using effective computation of invariants $k[V]^{G}$ for a finitely generated $G$-module $V$ $[14,27,58]$.

Lemma 3 Let $X$ be a quasiprojective normal variety over $k$, given by explicit equations, and let $U \subset X$ be a nonempty subvariety. Given $d \in \mathbb{N}$, there is an effective procedure to produce a finite extension $k^{\prime}$ of $k$ and a finite collection of normal quasiprojective varieties $Y^{(1)}, \ldots$, $Y^{(m)}$ over $k^{\prime}$, with finite morphisms $f_{i}: Y^{(i)} \rightarrow X_{k^{\prime}}$, such that for each $i$ the restriction of $f_{i}$ to $f_{i}^{-1}\left(U_{k^{\prime}}\right)$ is étale of degree $d$, and such that the $Y_{\bar{k}}^{(i)} \rightarrow X_{\bar{k}}(i=1, \ldots, m)$ are up to isomorphism all the degree d coverings by normal quasiprojective varieties over $\bar{k}$ that are étale on the pre-image of $U_{\bar{k}}$.

Proof Let $N=\operatorname{dim} X$. Shrinking $U$ if necessary we may suppose that there is a morphism $U \rightarrow \mathbb{P}^{N-1}$, given by a suitable linear projection, such that the generic fiber is smooth and one-dimensional, i.e., the restriction to the generic point $\eta=\operatorname{Spec}\left(k\left(x_{1}, \ldots, x_{N-1}\right)\right)$ (where the $x_{i}$ denote coordinates on a standard affine chart of $\mathbb{P}^{N-1}$ ) is a nonsingular quasiprojective curve $C_{\eta}$. We may effectively compute a nonsingular projective compactification $\bar{C}_{\eta}$. The genus $g=g\left(\bar{C}_{\eta}\right)$ and degree $e=\operatorname{deg}\left(\bar{C}_{\eta} \backslash C_{\eta}\right)$, together with $d$, determine by the Riemann-Hurwitz formula an upper bound $g_{\max }$ on the genus of $Y \times{ }_{X} \eta$. Using effective Hilbert scheme techniques we construct parameter spaces containing every isomorphism class of genus $g^{\prime} \leq g_{\max }$ curve equipped with a morphism of degree $d$ to $\bar{C}_{\eta}$. That the ramification divisor is contained in the scheme-theoretic pre-image of $\bar{C}_{\eta} \backslash C_{\eta}$ is a closed condition and one that may be implemented effectively (cf. [48, Corollary 3.14]) and determines a finite field extension $k^{\prime}$ of $k$ and a finite set of candidates for $Y \times_{X} \eta$, over $k^{\prime}$. Applying effective normalization to each of these candidates and eliminating those which are not étale over $U_{k^{\prime}}$, we obtain the $Y^{(i)}$.

\section{Baily-Borel compactifications}

Let $\mathbb{D}=G / K$ be a bounded symmetric domain and $\Gamma$ an arithmetic subgroup of $G$. It is known that $\Gamma \backslash \mathbb{D}$ admits a canonical compactification $(\Gamma \backslash \mathbb{D})^{*}$, the Baily-Borel compactification, which is a normal projective variety [5]; however, the construction of this variety does not supply algebraic equations. Let $\widetilde{\Gamma}<\Gamma$ be a finite-index subgroup, and assume that $\widetilde{\Gamma}$ is neat. (Recall that an arithmetic subgroup is called neat if, for every element, the subgroup of $\mathbb{C}^{*}$ generated by its eigenvalues is torsion-free.) In this section, we show that if we know $\Gamma \backslash \mathbb{D}$ as a quasiprojective variety (with explicit equations over some number field), we can effectively construct $\widetilde{\Gamma} \backslash \mathbb{D}$ as a quasiprojective variety (as one of finitely many candidates), together with its Baily-Borel compactification.

In the following, we let $k$ denote a number field.

Lemma 4 Let $G$ be a linear algebraic group over $k$ and $\Gamma$ an arithmetic subgroup. There is an effective procedure to construct a neat subgroup of finite index in $\Gamma$.

Proof It suffices to establish the result for a discrete subgroup of $G L_{n}(\mathbb{Z})$. Fix a prime $\ell$. There is a finite extension $\mathbf{k}$ of $\mathbb{Q}_{\ell}$ over which every polynomial of degree $n$ with coefficients in $\mathbb{Q}_{\ell}$ factors completely (see [32], effectively computed in [56]). The structure of $\mathbf{k}^{*}$ is known as a direct sum of $\mathbb{Z}$, a finite group, and $\mathbb{Z}_{\ell}^{N}$ for some $N$. Then there is an $\varepsilon>0$ 
such that ball of radius $\varepsilon$ around 1 is contained in the free part, so an $n \times n$ integer matrix sufficiently close $\ell$-adically to the identity matrix has its eigenvalues not more than $\varepsilon$ away from 1 . Hence they generate a torsion free subgroup of $\mathbf{k}^{*}$, and also of $\mathbb{C}^{*}$.

We fix an embedding $k \hookrightarrow \mathbb{C}$.

Proposition 5 Let $\mathbb{D}=G / K$ be a bounded symmetric domain, $\Gamma$ an arithmetic subgroup of $G$, and $\widetilde{\Gamma}$ a finite-index subgroup of $\Gamma$ which is neat. Let $X^{\circ}$ be a quasiprojective variety over $k$, given by explicit equations, and let $U \subset X^{\circ}$ be a nonempty open subscheme, also explicitly given. Suppose that there exists an isomorphism $X_{\mathbb{C}}^{\circ} \stackrel{\sim}{\rightarrow} \Gamma \backslash \mathbb{D}$ such that the map $\mathbb{D} \rightarrow \Gamma \backslash \mathbb{D}$ is unramified over the image of $U_{\mathbb{C}}$. Then there is an effective procedure to produce a finite extension $k^{\prime}$ of $k$ with compatible embedding in $\mathbb{C}$ and a finite collection of nonsingular quasiprojective varieties $Y^{(1)}, \ldots, Y^{(m)}$ defined over $k^{\prime}$ with morphisms $f_{i}: Y^{(i)} \rightarrow X_{k^{\prime}}^{\circ}$, such that, for some $i$, setting $\widetilde{X}^{\circ}:=Y^{(i)}$ there exists an isomorphism $\widetilde{X}_{\mathbb{C}}^{\circ} \stackrel{\sim}{\rightarrow} \widetilde{\Gamma} \backslash \mathbb{D}$ fitting into a commutative diagram

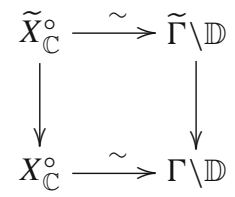

Proof Since $\widetilde{\Gamma} \backslash \mathbb{D} \rightarrow \Gamma \backslash \mathbb{D}$ ramifies over the same set of points as $\mathbb{D} \rightarrow \Gamma \backslash \mathbb{D}$, this follows directly from Lemma 3.

Proposition 6 Let $\mathbb{D}=G / K$ be a bounded symmetric domain, $\Gamma$ a neat arithmetic subgroup of $G$, and $X^{\circ}$ a quasiprojective variety over $k$ given by explicit equations such that $X_{\mathbb{C}}^{\circ}$ is isomorphic to $\Gamma \backslash \mathbb{D}$. Assume that $P G L_{2}$ is not a quotient of $G$. Then there is an effective procedure to construct a projective variety $X$ over $k$, together with open immersion $X^{\circ} \rightarrow X$, such that $X_{\mathbb{C}}$ is isomorphic to the Baily-Borel compactification $(\Gamma \backslash \mathbb{D})^{*}$.

The first ingredient in the proof of Proposition 6 is a result of Alexeev [1, §3], building on earlier work of Mumford [51]:

Theorem 7 Let $\mathbb{D}$ be a bounded Hermitian symmetric domain and $\Gamma$ a neat arithmetic subgroup acting on $\mathbb{D}$. Let $X^{\circ}=\Gamma \backslash \mathbb{D}$ with Baily-Borel compactification $X$ and boundary $\Delta$. Then $(X, \Delta)$ is log canonical, with the automorphic factor coinciding with the log canonical divisor $K_{X}+\Delta$.

We will also use a result of Fujino [17]:

Theorem 8 Let $(X, \Delta)$ be a projective log canonical pair and $M$ a line bundle on $X$. Assume that $M \equiv K_{X}+\Delta+N$, where $N$ is an ample $\mathbb{Q}$-divisor on $X$. Let $x_{1}, x_{2} \in X$ be closed points and assume there are positive numbers $c(k)$ with the following properties:

(1) If $Z \subset X$ is an irreducible (positive-dimensional) subvariety that contains $x_{1}$ or $x_{2}$ then

$$
\left(N^{\operatorname{dim}(Z)} \cdot Z\right)>c(\operatorname{dim}(Z))^{\operatorname{dim}(Z)} .
$$

(2) The numbers $c(k)$ satisfy the inequality

$$
\sum_{k=1}^{\operatorname{dim}(X)} \sqrt[k]{2} \frac{k}{c(k)} \leq 1 .
$$


Then the global sections of $M$ separate $x_{1}$ and $x_{2}$.

Proof of Proposition 6 The hypotheses guarantee that the complement of $\Gamma \backslash \mathbb{D}$ in the BailyBorel compactification has codimension $\geq 2$. If we define

$$
X=\operatorname{Proj}\left(\bigoplus_{d \geq 0} H^{0}\left(X^{\circ}, d K_{X^{\circ}}\right)\right) .
$$

then $X$ satisfies the conditions of the proposition. It remains to show that we can construct $X$ effectively.

Effective resolution of singularities as in Sect. 2 allows us to construct a nonsingular compactification $\widetilde{X}$ of $X^{\circ}$, projective, such that $\widetilde{X} \backslash X^{\circ}$ is a simple normal crossings divisor $D_{1} \cup \cdots \cup D_{m}$.

Now the Borel extension property [8, Thm. A] implies that the inclusion $X^{\circ} \rightarrow X$ extends to a birational morphism $\pi: \widetilde{X} \rightarrow X$. By Theorem $7, X$ has at worst log canonical singularities. Hence there are integers $c_{i} \leq 1$ such that

$$
\pi^{*} K_{X}=K_{\tilde{X}}+\sum c_{i} D_{i} .
$$

By the chain of inclusions

$$
H^{0}\left(\widetilde{X}, d\left(\pi^{*} K_{X}\right)\right) \subseteq H^{0}\left(\tilde{X}, d\left(K_{\tilde{X}}+\sum D_{i}\right)\right) \subseteq H^{0}\left(X^{\circ}, d K_{X^{\circ}}\right)
$$

we deduce that

$$
H^{0}\left(X, d K_{X}\right)=H^{0}\left(\tilde{X}, d\left(K_{\tilde{X}}+\sum D_{i}\right)\right) .
$$

Theorem 8 supplies a universal constant $n$ depending only on the dimension of $X$, such that for any $d \geq n$, the linear system $\left|d K_{X}\right|$ separates points on $X$. The image $X^{\prime}$ of $\left|d K_{X}\right|$ may be effectively computed using (3.1). The normalization of $X^{\prime}$, which may also be computed effectively, is then isomorphic to $X$.

Remark 9 There are examples in the literature in which $X^{\circ}$ as in Proposition 5 has been constructed. In each case, $\Gamma \backslash \mathbb{D}$ is a quotient of a period domain associated to the geometric objects being parametrized.

- Abelian varieties

- Polarized, with symmetric theta structure [50,53].

- Polarized, with level $n$ structure $(n \geq 3)$ : a construction based on Hilbert scheme and geometric invariant theory, presented in $[54, \S 7.3]$, can be carried out effectively by the techniques mentioned in Section 2.

- $K 3$ surfaces

- A six-dimensional ball quotient as a moduli space of $K 3$ surfaces which are cyclic degree 4 covers of $\mathbb{P}^{2}$ ramified along a quartic $[4,30]$.

- A nine-dimensional ball quotient coming from $K 3$ surfaces which are cyclic triple covers of $\mathbb{P}^{1} \times \mathbb{P}^{1}$ with branch curve of bidegree $(3,3)$ [31].

- K3 surfaces of degree 2, described by Horikawa [22] and Shah [61]; see also [39].

- A four-, resp. ten-dimensional ball quotient arising from the moduli space of cubic surfaces [2], resp. threefolds [3]. 
Mumford's construction in the abelian variety setting yields explicit equations for the moduli space together with a universal family. In each of the other examples, explicit GIT constructions are given, and from these we may obtain explicit equations as mentioned in Sect. 2. This allows us, e.g., to compute the point in $X^{\circ}$ corresponding to a given $K 3$ surface of degree 2 presented as a double cover of the plane branched along an explicitly given sextic curve.

Remark $10 \mathrm{~K} 3$ surfaces of degree 4 are analyzed in [38,62], and degrees up to 8 in [40], via the GIT of quartic surfaces, respectively complete intersections. The analysis yields a factorization of the rational map from the Baily-Borel compactification to the GIT quotient. It would be interesting to use this to give an effective construction of $X^{\circ}$ for these degrees generalizing the one for degree 2, which is based on an explicit weighted Kirwan blowup of the GIT quotient of plane sextics [28].

Remark 11 An effective construction of a Baily-Borel compactification is tantamount to effectively bounding degrees of generators of the corresponding ring of automorphic functions. The technique in [5] for proving the existence of projective compactifications is not effective as it relies on a compactness argument. In some examples these rings have been computed explicitly: Igusa [25] shows that the ring of Siegel modular forms for principally polarized abelian surfaces are generated by Eisenstein series of weights 4, 6, 10, and 12 . The case of threefolds is explored by Tsuyumine [67], who shows that 34 modular forms, of weights ranging from 4 to 48 , suffice. Not all of these may be expressed in terms of Eisenstein series. The case of fourfolds is addressed in Freitag-Oura [16], who introduce some specific relations and a dimension formula. Additional work in this direction was done by Oura-Poor-Yuen [55].

Proposition 12 Let $k$ be a number field with a given embedding in $\mathbb{C}$. Let $X$ and $X^{\prime}$ be projective varieties over $k$ satisfying $X_{\mathbb{C}} \cong(\Gamma \backslash \mathbb{D})^{*}$ and $X_{\mathbb{C}}^{\prime} \cong\left(\Gamma^{\prime} \backslash \mathbb{D}^{\prime}\right)^{*}$, and suppose that $\Gamma^{\prime}$ is neat. Fix an integer $d$. Then there is an effective procedure to produce a finite extension $k^{\prime}$ of $k$ with a compatible embedding in $\mathbb{C}$ and morphisms $f_{1}, \ldots, f_{m}: X_{k^{\prime}} \rightarrow X_{k^{\prime}}^{\prime}$ such that $\left(f_{1}\right)_{\mathbb{C}}, \ldots,\left(f_{m}\right)_{\mathbb{C}}$ are all the morphisms $X_{\mathbb{C}} \rightarrow X_{\mathbb{C}}^{\prime}$ under which the pullback of $K_{X_{\mathbb{C}}^{\prime}}$ is isomorphic to $d K_{X_{\mathbb{C}}}$.

Proof The Hilbert scheme representing morphisms of the given degree from $X$ to $X^{\prime}$ may be constructed effectively and by rigidity [49] has dimension zero.

\section{Abelian varieties}

Let $A$ be an abelian variety over a number field $k$.

Lemma 13 Assume that $A$ is given by explicit equations. Then there is an effective way to produce a finite extension $k^{\prime}$ of $k$ such that A acquires semistable reduction after base change to $k^{\prime}$.

Proof This is done by Proposition 4.7 of [19].

We recall two notions of heights of abelian varieties. The Faltings height is computed using a semistable model. Let $K$ be a finite extension of $k$ and $\mathcal{A}$ a semistable model over the ring of integers $\mathfrak{o}_{K}$. Then the Faltings height is the arithmetic degree of a particular metrized canonical sheaf on $\mathcal{A}$

$$
h_{F}(A)=\frac{1}{[K: \mathbb{Q}]} \widehat{\operatorname{deg}} \bar{\omega}_{\mathcal{A} / \operatorname{Spec}\left(\mathfrak{o}_{K}\right)}
$$


This is independent of the choices of $K$ and $\mathcal{A}$. For details see, e.g., [9, §2.1.3].

Alternatively, the theta height is defined purely algebraically, in terms of a principal polarization. In the following two effectivity results, the complexity is bounded explicitly in terms of $[k: \mathbb{Q}], \operatorname{dim} A$, and $h_{F}(A)$. Effective comparison results between $h_{F}(A)$ and the theta height are well known; see, e.g., [57].

Proposition 14 Let $A$ be a polarized abelian variety over $k$ defined by explicit equations. Then there is an effective procedure to compute a finite extension $k^{\prime}$ of $k$ for which we have

$$
\operatorname{End}_{k^{\prime}}(A)=\operatorname{End}_{\bar{k}}(A),
$$

as well as generators of the $\mathbb{Z}$-module $\operatorname{End}_{k^{\prime}}(A)$.

Proof To obtain $\operatorname{End}_{k^{\prime}}(A)=\operatorname{End}_{\bar{k}}(A)$ we first reduce to the case when $A$ has a semistable model over $k$ by effective semistable reduction (Lemma 13). There is an effective bound on [ $\left.k^{\prime}: k\right]$ from Lemma 2.1 of [42]. The minimal $k^{\prime}$ is unramified over $k$ by Theorem 1.3 of [59], hence (Hermite-Minkowski) suitable $k^{\prime}$ can be determined effectively.

The main result of [44] (see also [9]) bounds the discriminant of the ring of endomorphisms, which by positive-definiteness bounds the degree of the graphs of the elements in a $\mathbb{Z}$-basis of $\operatorname{End}_{k^{\prime}}(A)$ (cf. [7, Lemma 5.1.6, Corollary 5.1.7]). So they can be found effectively.

Proposition 15 ([46], Theorem 1) Let $A$ be an abelian variety over $k$. Then there exists an effective $M \in \mathbb{N}$ such that for any $m$, if we let $A_{m}$ denote the $m$-torsion subgroup of $A(\bar{k})$, then

$$
\operatorname{End}(A) \rightarrow \operatorname{End}_{\mathrm{Gal}(\bar{k} / k)}\left(A_{m}\right)
$$

has cokernel annihilated by $M$. In particular, for a prime $\ell \nmid M$ the natural homomorphism

$$
\operatorname{End}(A) / \ell \rightarrow \operatorname{End}_{\mathrm{Gal}(\bar{k} / k)}\left(A_{\ell}\right)
$$

is an isomorphism.

\section{Effective Kuga-Satake construction}

Let $k$ be a number field with an embedding in $\mathbb{C}$ and $d$ a positive integer.

Definition 16 A polarization (resp. quasi-polarization) of degree $2 d$ on a $K 3$ surface $S$ over $k$ is a Galois-invariant class in $\operatorname{Pic}\left(S_{\bar{k}}\right)$ that is ample (resp. nef) and has self-intersection $2 d$. A primitive polarization (or quasi-polarization) is one that is not a nontrivial multiple of another polarization (or quasi-polarization).

Remark 17 Suppose $S$ is given by explicit equations. These determine a very ample line bundle $L=\mathcal{O}_{S}(1)$. By standard Hilbert scheme techniques we can effectively determine whether the polarization $L$ is primitive, and when it is not, we can produce explicitly a finite extension $k^{\prime}$ of $k$ and a primitive polarization represented by a line bundle $L^{\prime}$ on $S_{k^{\prime}}$. If we assume, further, that $S\left(k_{v}\right) \neq \emptyset$ for all places $v$ of $k$, then a standard descent argument (see, e.g., $\$ 4$ of [34]) produces effectively a line bundle on $S$ whose base change to $S_{k^{\prime}}$ is isomorphic to $L^{\prime}$.

For the remainder of the paper we make the following assumptions. 
Assumption 18 We assume there is an effective construction of $X^{\circ}$ over $k$ with $X_{\mathbb{C}}^{\circ}$ isomorphic to the period space $\Gamma \backslash \mathbb{D}$ of primitively quasi-polarized $K 3$ surfaces of degree $2 d$. Given a $K 3$ surface $S$ over $k$ with explicit equations and supplied with an explicitly given ample polarizing class of degree $2 d$, we assume we can effectively produce the corresponding point in $X^{\circ}$.

Let $n$ be a positive integer, greater than or equal to 3 .

The Kuga-Satake construction has been treated in $[13,18,36,60]$. Here we follow the treatment in [60], where the relevant level structures are described explicitly and the result is the existence of morphisms

$$
f_{d, a, n, \gamma}^{k s}: \mathcal{F}_{2 d, n^{\mathrm{sp}}} \rightarrow \mathcal{A}_{g, d^{\prime}, n}
$$

of moduli spaces defined over an explicit number field. Here, there is a standard quadratic form $Q$ on the primitive $H^{2}$ lattice $P$ of the $K 3$ surface, whose even Clifford algebra will be denoted $C^{+}(P)$, and $a$ is an element of the opposite algebra $C^{+}(P)^{\text {op }}$ satisfying certain conditions. Then $d^{\prime}$ depends explicitly on $a$ and $d$, and $\gamma$ belongs to a nonempty finite index set. We suppose these choices are fixed. The morphism extends to a morphism of BailyBorel compactifications. The compactified source and target spaces can be constructed as projective varieties over an explicit number field (up to finitely many candidates) using Propositions 5 and 6 by the observations of Remark 9. Then (again up to finitely many candidates) Proposition 12 produces $f_{d, a, n, \gamma}^{k s}$.

The Kuga-Satake abelian variety $A$ associated to the polarized $K 3$ surface $S$ has the following characterization (cf. [18]).

Let $e_{1}, \ldots, e_{21}$ be linearly independent vectors in $P$ diagonalizing the quadratic form so that the span of $e_{1}$ and $e_{2}$ is negative-definite and the span of $e_{3}, \ldots, e_{21}$ is positive-definite. Let $f_{1}, f_{2} \in P_{\mathbb{R}}$ satisfy $f_{1}+i f_{2} \in P^{2,0}$ and $Q\left(f_{1}\right)=-1$. Then $f_{1}$ and $f_{2}$ determine an element

$$
J:=f_{1} f_{2} \in C^{+}(P)_{\mathbb{R}} .
$$

The element $J$ is independent of the choice of $f_{1}$ and $f_{2}$, and determines a complex structure on $C^{+}(P)_{\mathbb{R}}$.

The $\mathbb{C}^{*}$-action on $C^{+}(P)_{\mathbb{R}}$

$$
(a+b i) \cdot x:=(a-b J) x
$$

determines a Hodge structure of weight 1 on $C^{+}(P)$. For a suitable choice of sign \pm , the element $\alpha:= \pm e_{1} e_{2} \in C^{+}(P)$ and anti-involution $\iota: C^{+}(P) \rightarrow C^{+}(P)$,

$$
\iota\left(e_{i_{1}} \cdots e_{i_{m}}\right):=e_{i_{m}} \cdots e_{i_{1}}, \quad\left(i_{1}<\cdots<i_{m}\right),
$$

determine a polarization

$$
E: C^{+}(P) \times C^{+}(P) \rightarrow \mathbb{Z}, \quad(v, w) \mapsto \operatorname{tr}(\alpha \iota(v) w)
$$

where $\operatorname{tr}(c)$ denotes the trace of the map $x \mapsto c x$. Then the Kuga-Satake abelian variety associated with the polarized $K 3$ surface $S$ is

$$
A:=\left(C^{+}(P)_{\mathbb{R}}, J\right) / C^{+}(P),
$$

which is a complex torus with polarized Hodge structure, i.e., a polarized abelian variety over $\mathbb{C}$. 
The following properties hold. There is an injective ring homomorphism

$$
u: C^{+}(P) \rightarrow \operatorname{End}\left(H^{1}(A, \mathbb{Z})\right)
$$

compatible with the weight zero Hodge structures on source and target. The abelian variety $A$ will be defined over a number field, and the homomorphism of $\mathbb{Z}_{\ell}$-modules obtained from $u$ is a homomorphism of Galois modules.

\section{Computing the Picard group of a $K 3$ surface}

From the Kuga-Satake construction we get an injective homomorphism of Hodge structures

$$
\operatorname{End}\left(C^{+}(P)\right)_{\mathbb{Q}} \cong C^{+}(P)_{\mathbb{Q}}^{*} \otimes C^{+}(P)_{\mathbb{Q}}^{*} \rightarrow H^{2}(A \times A, \mathbb{Q})
$$

The intersection of the $(0,0)$-part of $\operatorname{End}\left(C^{+}(P)\right)_{\mathbb{C}}$ with $\operatorname{End}\left(C^{+}(P)\right)_{\mathbb{Q}}$ may be effectively computed by identifying End $\left(C^{+}(P)\right)_{\mathbb{Q}}$ with a direct summand of $H^{2}(A \times A, \mathbb{Q})$. This will permit us to reduce the computation of the Picard group of a $K 3$ surface to the determination of the Néron-Severi group of a polarized abelian variety.

Proposition 19 Let $S$ be a $K 3$ surface as in Assumption 18. Then there is an effective procedure to compute $\operatorname{Pic}\left(S_{\bar{k}}\right)$ by means of generators with explicit equations over a finite extension of $k$.

Remark 20 We are interested in the computation of $H_{*}(X(\mathbb{C}), \mathbb{Z})$, where $X$ is a smooth projective variety over $k$. This can be done effectively, as explained in [68], by embedding $X(\mathbb{C})$ in a Euclidean space, subdividing the Euclidean space into cubes, and intersecting with $X(\mathbb{C})$. When $\operatorname{dim} X=2$ this has been treated in [33]. When $X(\mathbb{C})$ is isomorphic to a quotient $\mathbb{C}^{N} / \Lambda$ for some $N$ and lattice $\Lambda \subset \mathbb{C}^{N}$ this is known and standard.

Proof of Proposition 19 By the assumptions, we may choose a lift in $\mathcal{F}_{2 d, n^{\text {sp }}}$ of the moduli point of $S$, and hence obtain a finite set of candidates for the Kuga-Satake abelian variety. We compute $P \subset H^{2}(S(\mathbb{C}), \mathbb{Z})$ and $E: C^{+}(P) \times C^{+}(P) \rightarrow \mathbb{Z}$ exactly and $J \in C^{+}(P)_{\mathbb{R}}$ to high precision. The convergence of theta functions is well understood (cf. [52, §2.1]), so we may, by evaluating theta functions to high enough precision, identify the correct image point of the Kuga-Satake morphism, let us say $A^{k s}$ defined over a finite extension of $k$, and thereby obtain an analytic map $\mathbb{C}^{2^{19}} \rightarrow A^{k s}(\mathbb{C})$ giving rise to $A \stackrel{\sim}{\rightarrow} A^{k s}(\mathbb{C})$, to arbitrarily high precision. Using Proposition 14 we compute representatives for generators of $\operatorname{NS}\left(A_{\mathbb{C}}^{k s} \times A_{\mathbb{C}}^{k s}\right) \otimes \mathbb{Q}$ under the standard identification with $\operatorname{End}^{s y m}\left(A_{\mathbb{C}}^{k s} \times A_{\mathbb{C}}^{k s}\right) \otimes \mathbb{Q}(\mathrm{cf} .[7, \S 5.2])$, noting that denominators are controlled by the degree of the polarization. So, computation to sufficiently high precision determines classes spanning $H^{2}(A \times A, \mathbb{Q})$. This determines the classes of type $(0,0)$ in $\operatorname{End}\left(C^{+}(P)\right)_{\mathbb{Q}}$. By $v \mapsto\left(y \mapsto v y e_{1}\right)$ with the notation of the previous section, we have an injection

$$
P \rightarrow \operatorname{End}\left(C^{+}(P)\right)_{\mathbb{Q}},
$$

as described in [18, Proposition 6.3], therefore by computing the pre-image the $(0,0)$-classes in $P$ are determined as well. These classes and the polarizing class span the image of $\operatorname{Pic}\left(S_{\bar{k}}\right) \otimes$ $\mathbb{Q}$ in $H^{2}(S(\mathbb{C}), \mathbb{Q})$, hence by intersecting with $H^{2}(S(\mathbb{C}), \mathbb{Z})$ and using the fact that $\pm D$ is effective for any divisor class $D$ on a $K 3$ surface with self-intersection $\geq-2$ (consequence of Riemann-Roch) we easily obtain a degree bound on generators of $\operatorname{Pic}\left(S_{\bar{k}}\right)$ represented by effective divisors on $S_{\bar{k}}$. Using Hilbert schemes we may obtain representatives of the effective 
divisor classes in all degrees up to this bound, each given by explicit equations, defined over a finite extension of $k$.

\section{Proof for good primes}

We keep the notation of the previous section and let $\Gamma=\operatorname{Gal}(\bar{k} / k)$.

Proposition 21 Let $S$ be a $K 3$ surface as in Assumption 18. Then there exists, effectively, an $\ell_{0}$ such that for all primes $\ell>\ell_{0}$ we have $\operatorname{Br}\left(S_{\bar{k}}\right)_{\ell}^{\Gamma}=0$.

The rest of this section is devoted to the proof. Proposition 19 tells us that after suitably extending $k$ we may suppose that $\operatorname{Pic}\left(S_{\bar{k}}\right)$ is defined over $k$ and is known explicitly. We suppose also that we have obtained the Kuga-Satake abelian variety $A^{k s}$ with analytic map $\underset{\rightarrow}{\sim} A^{k s}(\mathbb{C})$ that can be computed to arbitrarily high precision, and that $\operatorname{End}\left(A_{\bar{k}}^{k s}\right)$ is defined over $k$ and is known explicitly. Then we may suppose that the subalgebra of $\operatorname{End}\left(H_{1}(A, \mathbb{Z})\right)$ corresponding to endomorphisms of $A^{k s}$ has been identified, i.e., that we have computed

$$
\operatorname{End}(A) \subset \operatorname{End}\left(H_{1}(A(\mathbb{C}), \mathbb{Z})\right) .
$$

We have the exact sequence (cf. equation (5) of [65])

$$
\begin{aligned}
0 & \rightarrow \operatorname{Pic}(S) / \ell^{n} \rightarrow H^{2}\left(S_{\bar{k}}, \mu \ell^{n}\right)^{\Gamma} \rightarrow \operatorname{Br}\left(S_{\bar{k}}\right)_{\ell^{n}} \\
& \rightarrow H^{1}\left(k, \operatorname{Pic}\left(S_{\bar{k}}\right) / \ell^{n}\right) \rightarrow H^{1}\left(k, H^{2}\left(S_{\bar{k}}, \mu_{\ell^{n}}\right)\right) .
\end{aligned}
$$

Let $\delta$ be the (absolute value of the) discriminant of the Néron-Severi group. Then there is an exact sequence

$$
0 \rightarrow \operatorname{Pic}(S) \oplus T_{S} \rightarrow H^{2}(S(\mathbb{C}), \mathbb{Z}) \rightarrow K \rightarrow 0
$$

where the cokernel $K$ is finite, of order $\delta$. After tensoring with $\mathbb{Z}_{\ell}$ and using a comparison theorem this becomes an exact sequence of Galois modules

$$
0 \rightarrow\left(\operatorname{Pic}(S) \otimes \mathbb{Z}_{\ell}\right) \oplus T_{S, \ell} \rightarrow H^{2}\left(S_{\bar{k}}, \mathbb{Z}_{\ell}(1)\right) \rightarrow K_{\ell} \rightarrow 0
$$

for some finite $K_{\ell}$, where $T_{S, \ell}$ is the submodule of $H^{2}\left(S_{\bar{k}}, \mathbb{Z}_{\ell}(1)\right)$ orthogonal to $\operatorname{Pic}(S)$; note that as abelian groups, $T_{S, \ell} \cong T_{S} \otimes \mathbb{Z}_{\ell}$ and $K_{\ell} \cong K \otimes \mathbb{Z}_{\ell}$. In particular, for $\ell \nmid \delta$ and any $n$ we have

$$
H^{1}\left(k, \operatorname{Pic}(S) / \ell^{n}\right) \hookrightarrow H^{1}\left(k, H^{2}\left(S_{\bar{k}}, \mu_{\ell^{n}}\right)\right),
$$

and the 5-term exact sequence reduces to an isomorphism

$$
\left(T_{S, \ell} / \ell^{n}\right)^{\Gamma} \stackrel{\sim}{\rightarrow} \operatorname{Br}\left(S_{\bar{k}}\right)_{\ell^{n}}^{\Gamma}
$$

Applying transpose to the homomorphism $u$ of (5.1) we get a homomorphism

$$
\text { tu }: C^{+}(P) \rightarrow \operatorname{End}\left(H_{1}(A, \mathbb{Z})\right) .
$$

If $\operatorname{Pic}(S)$ has rank at least 2, then we let $m \in P$ be an algebraic class and construct

$$
m \wedge T_{S} \subset \operatorname{End}\left(H_{1}(A(\mathbb{C}), \mathbb{Z})\right) .
$$

By consideration of Hodge type, End $(A)$ and $m \wedge T_{S}$ are disjoint in $\operatorname{End}\left(H_{1}(A(\mathbb{C}), \mathbb{Z})\right)$. Now, outside of finitely many $\ell$ (effectively) we have an injective map

$$
\operatorname{End}(A) / \ell \rightarrow \operatorname{End}\left(H_{1}(A(\mathbb{C}), \mathbb{Z})\right) / \ell
$$


coming from (7.1) and injective composite map

$$
T_{S} / \ell \rightarrow\left(m \wedge T_{S}\right) / \ell \rightarrow \operatorname{End}\left(H_{1}(A(\mathbb{C}), \mathbb{Z})\right) / \ell,
$$

such that the images in $\operatorname{End}\left(H_{1}(A(\mathbb{C}), \mathbb{Z})\right) / \ell$ are disjoint.

We use the natural isomorphism of Galois modules

$$
A_{\ell}^{k s} \cong \operatorname{Hom}\left(H^{1}\left(A^{k s}, \mathbb{Z} / \ell\right), \mathbb{Z} / \ell\right)
$$

(cf. [65, §4.1]) and view $\left(m \wedge T_{S, \ell}\right) / \ell$ as a subgroup of $\operatorname{End}\left(A_{\ell}^{k s}\right)$. So, we have an injective homomorphism of Galois modules

$$
T_{S, \ell} / \ell \rightarrow \operatorname{End}\left(A_{\ell}^{k s}\right) .
$$

Applying Proposition 15 we have, away from an effectively determined finite set of primes $\ell$, an isomorphism

$$
\operatorname{End}\left(A^{k s}\right) / \ell \underset{\sim}{\sim} \operatorname{End}_{\Gamma}\left(A_{\ell}^{k s}\right) .
$$

We conclude, outside of an effectively determined finite set of primes $\ell$, we have

$$
\left(T_{S, \ell} / \ell\right)^{\Gamma}=0 .
$$

If $\operatorname{Pic}(S)$ has rank one, then we have $T_{S}=P$, and we repeat the above argument using $\wedge^{20} T_{S}$ in place of $m \wedge T_{S}$ and an identification of $T_{S} / \ell$ with $\left(\wedge^{20} T_{S}\right) / \ell$ coming from $\wedge^{21} T_{S}$ $\cong \mathbb{Z}$.

\section{Bad primes}

Here we refine the arguments of Sect. 7 to get an effective bound on $\operatorname{Br}(S) / \operatorname{Br}(k)$. We treat the primes excluded from consideration in Sect. 7 one at a time, obtaining for each such prime $\ell$ an effective bound on the order of the $\ell$-primary subgroup of the image in $\operatorname{Br}\left(S_{\bar{k}}\right)$ of $\operatorname{Br}(S)$. As in the previous section, we extend $k$ and assume that $\operatorname{Pic}\left(S_{\bar{k}}\right)$ is defined over $k$ and the Kuga-Satake abelian variety together with its full ring of geometric endomorphisms is defined over $k$. We let $m$ be an integer such that the group $K_{\ell}$ of (7.2) is $\ell^{m}$-torsion and further extend $k$ so that the group $\operatorname{Br}\left(S_{\bar{k}}\right) \ell^{m}$ is defined over $k$. By [35] such a field extension may be produced effectively. To obtain an effective bound on the order of the $\ell$-primary subgroup of $\operatorname{Br}(S)$ in $\operatorname{Br}\left(S_{\bar{k}}\right)$ it suffices to produce an effective bound on the order of the cokernel of

$$
\operatorname{Pic}(S) / \ell^{n} \rightarrow H^{2}\left(S_{\bar{k}}, \mu_{\ell^{n}}\right)^{\operatorname{Gal}\left(\bar{k} / k^{\prime}\right)}
$$

that is independent of $n$.

The analysis of the previous section, in a refined form, yields an effective bound for $\left(T_{S, \ell} / \ell^{n}\right)^{\Gamma}$, independent of $n$. Indeed, Proposition 15 yields the effective annihilation of the cokernel of $\operatorname{End}\left(A^{k s}\right) \rightarrow \operatorname{End}_{\Gamma}\left(A_{\ell^{n}}^{k s}\right)$. In the portions of the argument where an injective homomorphism of finitely generated abelian groups is tensored with $\mathbb{Z} / \ell$, we obtain bounds independent of $n$ on the kernel of the homomorphism tensored with $\mathbb{Z} / \ell^{n}$, rather than injective homomorphisms. This suffices for the analysis.

Equation (1) of [65] yields an exact sequence of Galois modules

$$
0 \rightarrow \operatorname{Pic}(S) / \ell^{m} \rightarrow H^{2}\left(S_{\bar{k}}, \mu_{\ell^{m}}\right) \rightarrow \operatorname{Br}\left(S_{\bar{k}}\right) \ell^{m} \rightarrow 0,
$$

and therefore $\operatorname{Gal}\left(\bar{k} / k^{\prime}\right)$ acts trivially on $H^{2}\left(S_{\bar{k}}, \mu_{\ell}\right)$. By considering the sequence (7.2) tensored by $\mathbb{Z} / \ell^{m} \mathbb{Z}$ it follows that $\operatorname{Gal}\left(\bar{k} / k^{\prime}\right)$ acts trivially on $K_{\ell}$. 
We consider $n \geq m$ in what follows. Tensoring (7.2) with $\mathbb{Z} / \ell^{n}$ yields a four-term exact sequence of Galois modules with one Tor term:

$$
0 \rightarrow K_{\ell} \rightarrow \operatorname{Pic}(S) / \ell^{n} \oplus T_{S, \ell} / \ell^{n} \rightarrow H^{2}\left(S_{\bar{k}}, \mu_{\ell^{n}}\right) \rightarrow K_{\ell} \rightarrow 0 .
$$

Since $T_{S, \ell} / \ell^{n} \rightarrow H^{2}\left(S_{\bar{k}}, \mu_{\ell^{n}}\right)$ is injective, it follows that

$$
K_{\ell} \rightarrow \operatorname{Pic}(S) / \ell^{n}
$$

is injective.

We split the exact sequence (8.1) into two short exact sequences

$$
\begin{aligned}
& 0 \rightarrow K_{\ell} \rightarrow \operatorname{Pic}(S) / \ell^{n} \oplus T_{S, \ell} / \ell^{n} \rightarrow C \rightarrow 0, \\
& 0 \rightarrow C \rightarrow H^{2}\left(S_{\bar{k}}, \mu_{\ell^{n}}\right) \rightarrow K_{\ell} \rightarrow 0 .
\end{aligned}
$$

This gives the long exact sequences of Galois cohomology

$$
\begin{gathered}
K_{\ell} \hookrightarrow \operatorname{Pic}(S) / \ell^{n} \oplus\left(T_{S, \ell} / \ell^{n}\right)^{\Gamma} \rightarrow C^{\Gamma} \rightarrow H^{1}\left(\Gamma, K_{\ell}\right) \rightarrow H^{1}\left(\Gamma, \operatorname{Pic}(S) / \ell^{n} \oplus T_{S, \ell} / \ell^{n}\right), \\
0 \rightarrow C^{\Gamma} \rightarrow H^{2}\left(S_{\bar{k}}, \mu_{\ell^{n}}\right)^{\Gamma} \rightarrow K_{\ell} \rightarrow H^{1}(\Gamma, C) .
\end{gathered}
$$

Since (8.2) is an injective homomorphism of trivial Galois modules, the first three terms of the top sequence split off as a short exact sequence

$$
0 \rightarrow K_{\ell} \rightarrow \operatorname{Pic}(S) / \ell^{n} \oplus\left(T_{S, \ell} / \ell^{n}\right)^{\Gamma} \rightarrow C^{\Gamma} \rightarrow 0 .
$$

We conclude by calculating that

$$
\frac{\left|H^{2}\left(S_{\bar{k}}, \mu_{\ell^{n}}\right)^{\Gamma}\right|}{\left|\operatorname{Pic}(S) / \ell^{n}\right|} \leq \frac{\left|K_{\ell}\right| \cdot\left|C^{\Gamma}\right|}{\left|\operatorname{Pic}(S) / \ell^{n}\right|}=\left|\left(T_{S, \ell} / \ell^{n}\right)^{\Gamma}\right|,
$$

which is bounded as explained above.

Acknowledgments The authors would like to thank Nick Katz and Gisbert Wüstholz for helpful discussions.

\section{References}

1. Alexeev, V.: Log canonical singularities and complete moduli of stable pairs. Preprint (1996)

2. Allcock, D., Carlson, J.A., Toledo, D.: The complex hyperbolic geometry of the moduli space of cubic surfaces. J. Algebraic Geom. 11(4), 659-724 (2002)

3. Allcock, D., Carlson, J.A., Toledo, D.: The moduli space of cubic threefolds as a ball quotient. Mem. Amer. Math. Soc. 209(985) (2011)

4. Artebani, M.: A compactification of $\mathcal{M}_{3}$ via $K 3$ surfaces. Nagoya Math. J. 196, 1-26 (2009)

5. Baily, Jr., W.L., Borel, A.: Compactification of arithmetic quotients of bounded symmetric domains. Ann. Math. (2) 84(3), 442-528 (1966)

6. Bierstone, E., Grigoriev, D., Milman, P., Włodarczyk, J.: Effective Hironaka resolution and its complexity. Asian J. Math. 15(2), 193-228 (2011)

7. Birkenhake, C., Lange, H.: Complex abelian varieties, 2nd edn. Springer-Verlag, Berlin (2004)

8. Borel, A.: Some metric properties of arithmetic quotients of symmetric spaces and an extension theorem. J. Diff. Geom. 6, 543-560 (1972)

9. Bost, J.-B.: Périodes et isogenies des variétés abéliennes sur les corps de nombres (d'après D. Masser et G. Wüstholz). Séminaire Bourbaki 1994/95, Exp. No. 795. Asterisque 237, 115-161 (1996)

10. Bright, M.: Brauer groups of diagonal quartic surfaces. J. Symbolic Comput. 41(5), 544-558 (2006)

11. Charles, F.: On the Picard number of $K 3$ surfaces over number fields. Algebra \& Number Theory (to appear)

12. Colliot-Thélène, J.-L., Skorobogatov, A.N.: Descente galoisienne sur le groupe de Brauer. J. Reine Angew. Math. (to appear)

13. Deligne, P.: La conjecture de Weil pour les surfaces K3. Invent. Math. 15, 206-226 (1972) 
14. Derksen, H.: Computation of invariants for reductive groups. Adv. Math. 141, 366-384 (1999)

15. Faltings, G.: Endlichkeitssätze für abelsche Varietäten über Zahlkörpern. Invent. Math. 73(3), 349-366 (1983)

16. Freitag, E., Oura, M.: A theta relation in genus 4. Nagoya Math. J. 161, 69-83 (2001)

17. Fujino, O.: Effective base point free theorem for log canonical pairs, II: Angehrn-Siu type theorems. Michigan Math. J. 59(2), 303-312 (2010)

18. van Geemen, B.: Kuga-Satake varieties and the Hodge conjecture. In: The Arithmetic and Geometry of Algebraic Cycles (Banff, AB, 1998). NATO Sci. Ser. C Math. Phys. Sci., vol. 548, pp. 51-82. Kluwer Acad. Publ., Dordrecht (2000)

19. Grothendieck, A.: Modèles de Néron et monodromie. In: Groupes de monodromie en géométrie algébrique (SGA 7), I. Lect. Notes in Math., vol. 288. pp. 313-523, Springer-Verlag, Berlin (1972)

20. Hassett, B., Várilly-Alvarado, A., Varilly, P.: Transcendental obstructions to weak approximation on general K3 surfaces. Adv. Math. 228(3), 1377-1404 (2011)

21. Hoa, L.T.: Finiteness of Hilbert functions and bounds for Castelnuovo-Mumford regularity of initial ideals. Trans. Amer. Math. Soc. 360(9), 4519-4540 (2008)

22. Horikawa, E.: Surjectivity of the period map of $K 3$ surfaces of degree 2. Math. Ann. 228(2), 113-146 (1977)

23. Ieronymou, E.: Diagonal quartic surfaces and transcendental elements of the Brauer group. J. Inst. Math. Jussieu 9(4), 769-798 (2010)

24. Ieronymou, E., Skorobogatov, A.N., Zarhin, Y.G.: On the Brauer group of diagonal quartic surfaces. J. London Math. Soc. 83(3), 659-672 (2011)

25. Igusa, J.: On Siegel modular forms of genus two. Amer. J. Math. 84, 175-200 (1962)

26. Inose, H.: Defining equations of singular $K 3$ surfaces and a notion of isogeny. In: Proceedings of the international symposium on algebraic geometry (Kyoto, 1977), pp. 495-502. Kinokuniya Book Store, Tokyo (1978).

27. Kempf, G.R.: Computing invariants. In: Invariant Theory. Lect. Notes in Math., vol. 1278, pp. 81-94. Springer-Verlag, Berlin (1987)

28. Kirwan, F., Lee, R.: The cohomology of moduli spaces of $K 3$ surfaces of degree 2. I. Topology 28(4), 495-516 (1989)

29. Kleiman, S.: Les théorèmes de finitude pour le foncteur de Picard. In: Théorie des intersections et théorème de Riemann-Roch (SGA 6). Lect. Notes in Math., vol. 225. pp. 616-666, Springer-Verlag, Berlin (1971)

30. Kondō, S.: A complex hyperbolic structure for the moduli space of curves of genus three. J. Reine Angew. Math. 525, 219-232 (2000)

31. Kondō, S.: The moduli space of curves of genus 4 and Deligne-Mostow's complex reflection groups. In: Algebraic Geometry 2000, Azumino (Hotaka). Adv. Stud. Pure Math., vol. 36, pp. 383-400. Mathematical Society of Japan, Tokyo (2002)

32. Krasner, M.: Nombre des extensions d'un degré donné d'un corps p-adique. In: Les tendances géométriques en algèbre et théorie des nombres (Clemont-Ferrand, 1964), pp. 143-169. CNRS, Paris (1966)

33. Kresch, A.: CW complexes for complex algebraic surfaces. Exp. Math. 19(4), 413-419 (2010)

34. Kresch, A., Tschinkel, Y.: Effectivity of Brauer-Manin obstructions. Adv. Math. 218(1), 1-27 (2008)

35. Kresch, A., Tschinkel, Y.: Effectivity of Brauer-Manin obstructions on surfaces. Adv. Math. 226(5), 4131-4144 (2011)

36. Kuga, M., Satake, I.: Abelian varieties attached to polarized K3-surfaces. Math. Ann. 169, 239-242 (1967)

37. Kunyavskiı̆, B.È., Skorobogatov, A.N., Tsfasman, M.A.: Del Pezzo surfaces of degree four. Mém. Soc. Math. France (N.S.) 37 (1989)

38. Looijenga, E.: On quartic surfaces in projective 3-space. In: Proceedings, Bicentennial Congress Wiskundig genootschap (Amsterdam, 1978). Math. Centre Tracts, vol. 101, part II, pp. 269-274. Mathematica Centrum, Amsterdam (1979)

39. Looijenga, E.: New compactifications of locally symmetric varieties. In: Proceedings of the 1984 Vancouver Conference in Algebraic Geometry. CMS Conf. Proc., vol. 6, pp. 341-364. American Mathematical Socirty, Providence (1986).

40. Looijenga, E.: Compactifications defined by arrangements, II: Locally symmetric varieties of type IV. Duke Math. J. 119(3), 527-588 (2003)

41. van Luijk, R.: K3 surfaces with Picard number one and infinitely many rational points. Algebra \& Number Theory 1(1), 1-15 (2007)

42. Masser, D., Wüstholz, G.: Periods and minimal abelian subvarieties. Ann. Math. (2) 137(2), 407-458 (1993) 
43. Masser, D., Wüstholz, G.: Isogeny estimates for abelian varieties, and finiteness theorems. Ann. Math. (2) 137(3), 459-472 (1993)

44. Masser, D., Wüstholz, G.: Endomorphism estimates for abelian varieties. Math. Z. 215(4), 641-653 (1994)

45. Masser, D., Wüstholz, G.: Factorization estimates for abelian varieties. Inst. Hautes Études Sci. Publ. Math. 81, 5-24 (1995)

46. Masser, D., Wüstholz, G.: Refinements of the Tate conjecture for abelian varieties. In: Abelian varieties (Egloffstein, 1993), pp. 211-223. de Gruyter, Berlin (1995)

47. Mesnager, S.: Construction of the integral closure of an affine domain in a finite field extension of its quotient field. J. Pure Appl. Algebra 194(3), 311-327 (2004)

48. Mochizuki, S.: The geometry of the compactification of the Hurwitz scheme. Publ. Res. Inst. Math. Sci. 31(3), 355-441 (1995)

49. Mok, N.: Metric rigidity theorems on Hermitian locally symmetric manifolds. World Scientific Publishing Co., Teaneck (1989)

50. Mumford, D.: On the equations defining abelian varieties III. Invent. Math. 3, 215-244 (1967)

51. Mumford, D.: Hirzebruch's proportionality theorem in the non-compact case. Invent. Math. 42, 239-272 (1977)

52. Mumford, D.: Tata lectures on theta, I. Progr. Math., vol. 28. Birkhäuser Boston, Boston (1983)

53. Mumford, D.: Tata lectures on theta, III. Progr. Math., vol. 97. Birkhäuser Boston, Boston (1991)

54. Mumford, D., Fogarty, J., Kirwan, F.: Geometric invariant theory, 3rd edn. Springer-Verlag, Berlin (1994)

55. Oura, M., Poor, C., Yuen, D.S.: Towards the Siegel ring in genus four. Int. J. Number Theory 4(4), 563-586 (2008)

56. Pauli, S., Roblot, X.-F.: On the computation of all extensions of a $p$-adic field of a given degree. Math. Comp. 70(236), 1641-1659 (2001)

57. Pazuki, F.: Theta height and Faltings height. Bull. Soc. Math. France 140(1), 19-49 (2012)

58. Popov, V.L.: Constructive invariant theory. In: Young Tableaux and Schur Functors in Algebra and Geometry (Toruń, 1980). Astérisque, vol. 87-88, pp. 303-334. French Mathematical Society, Paris (1981)

59. Ribet, K.A.: Endomorphisms of semi-stable abelian varieties over number fields. Ann. Math. (2) 101(3), 555-562 (1975).

60. Rizov, J.: Kuga-Satake abelian varieties of $K 3$ surfaces in mixed characteristic. J. Reine Angew. Math. 648, 13-67 (2010)

61. Shah, J.: A complete moduli space for $K 3$ surfaces of degree 2. Ann. Math. (2) 122(3), 485-510 (1980)

62. Shah, J.: Degenerations of $K 3$ surfaces of degree 4. Trans. Amer. Math. Soc. 263(2), 271-308 (1981)

63. Shioda, T., Inose, H.: On singular $K 3$ surfaces. In: Complex analysis and algebraic geometry, pp. 119-136. Iwanami Shoten, Tokyo (1977)

64. Skorobogatov, A., Swinnerton-Dyer, P.: 2-descent on elliptic curves and rational points on certain Kummer surfaces. Adv. Math. 198(2), 448-483 (2005)

65. Skorobogatov, A.N., Zarhin, Y.G.: A finiteness theorem for the Brauer group of abelian varieties and $K 3$ surfaces. J. Algebraic Geom. 17(3), 481-502 (2008)

66. Swinnerton-Dyer, P.: Arithmetic of diagonal quartic surfaces, II. Proc. London Math. Soc. (3) 80(3), 513-544 (2000)

67. Tsuyumine, S.: On Siegel modular forms of degree three. Amer. J. Math. 108(4), 755-862, 1001-1003 (1986)

68. Whitney, H.: Geometric integration theory. Princeton Univ. Press, Princeton (1957)

69. Wittenberg, O.: Transcendental Brauer-Manin obstruction on a pencil of elliptic curves. In: Arithmetic of Higher-Dimensional Algebraic Varieties (Palo Alto, CA, 2002). Progr. Math., vol. 226, pp. 259-267. Birkhäuser Boston, Boston, MA (2004) 